\title{
Paraneoplastic Myoclonus with Papillary Thyroid Carcinoma
}

\author{
Hrayr Attarian $^{\mathrm{a}}$ Garrick Applebee $^{\mathrm{a}} \quad$ Audrey von Lepel $^{\mathrm{b}}$ \\ ${ }^{a}$ Vermont Regional Sleep Center, Burlington, Vt., and ${ }^{\mathrm{b}}$ Fairfax Associates in Medicine, Fairfax, Vt., USA
}

Dear Sir,

Myoclonus consists of sudden, involuntary muscular contractions [1]. The feature that distinguishes spinal, or segmental myoclonus from other forms of more generalized myoclonia is its restriction to one somatic region secondary to spinal cord pathology at the involved level [2]. Propriospinal myoclonus is characterized by generalized and symmetric jerks that arise from axial muscles and spread to other myotomes by means of propriospinal pathways [3].

Neurologic paraneoplastic disorders are syndromes that are neither due to direct metastases nor to toxicity of cancer therapy, coagulopathy, infection, or toxic/ metabolic causes. They are far less common than metastases and other nonmetastatic neurologic complications of cancer, but they are important because of the severe and permanent neurologic morbidity. Also, because often they are the presenting feature of an otherwise undiagnosed tumor, early diagnosis maximizes the likelihood of successful tumor treatment and favorable neurologic outcome [4].

\section{Case Report}

A 42-year-old woman presented with a 2-year history of primarily large amplitude jerks that had become a daily occurrence for the past 3 months, always involving the lower extremities, frequently the upper, and sometimes the torso. The movements were preceded by a sensation of an electric current shooting down her body, lasting 3-5 s and occurring every minute for up to $40 \mathrm{~min}$. A videotape of one such spell showed frequent myoclonic jerks affecting her lower extremities more than her upper extremities. She had them occasionally while standing up, but they never caused her to fall. She did not report loss of consciousness and had no history of seizures. There was no oral trauma or urinary incontinence with these episodes. She did have a 10-year history of restless leg syndrome and was being treated with clonazepam and trazodone. When these myoclonic jerks developed, she was also started on ropinirole with modest, if any, improvement in her symptoms.

The patient denied head trauma, stating that 20 years ago she had had a mild whip lash injury. She denied any family history of similar problems.

An EEG with added surface EMG electrodes applied to the tibialis anterior muscle revealed multiple jerks with no associated epileptiform abnormalities. An SSEP revealed normal latencies and no giant potentials. A previously performed CT scan of the brain was normal, and MRI of the cervical and thoracic spine was ordered. It did not show any neurological abnormalities, but did find an incidental solid enhancing lesion within the right lobe of the thyroid measuring approximately $2 \mathrm{~cm}$ in diameter. This appeared to be a solitary nodule and further evaluation with ultrasound and biopsy revealed papillary carcinoma. She underwent complete thyroidec- tomy and radiation therapy and was placed on thyroid hormone replacement with subsequent improvement in her myoclonus; she stated that they occurred less frequently, were much less intense and lasted a very short time. After tapering off her other medications and being placed on levetiracetam $500 \mathrm{mg}$ twice a day, she reported further modest improvement as well. We ordered amphiphysin antibody levels that were negative. We also ordered a full paraneoplastic antibody panel including CRMP-5-IgG, antineuronal nuclear antibody types 1-3, Purkinje cell cytoplasmic ab types 1, 2 and tr; $\mathrm{N}$ and $\mathrm{P} / \mathrm{Q}$ type calcium channel ab and AChR ganglionic neuronal ab levels all of which were negative.

\section{Discussion}

We report the first case of paraneoplastic propriospinal myoclonus due to papillary thyroid carcinoma. The fact that this preceded the discovery of the tumor by 2 years could be due to the slow growing nature of her neoplasm.

After thyroidectomy and radiation therapy, her symptoms improved significantly and were subsequently completely controlled with low-dose levetiracetam.

Although no paraneoplastic autoantibodies are specifically associated with papillary thyroid carcinoma, we planned to check for amphiphysin antibodies since they have been reported in other paraneoplastic myoclonus syndromes [5], but due

\section{KARGER}

Fax +4161306 1234 E-Mail karger@karger.ch www.karger.com
(C) 2007 S. Karger AG, Base 0014-3022/07/0583-0182\$23.50/0

Accessible online at: www.karger.com/ene
Hrayr Attarian

111 Colchester Avenue

Burlington, VT 05401 (USA)

Tel. +1 802847 5338, Fax +1 8028475679

E-Mail hattaria@uvm.edu 
to the cost involved the subject declined. The EEG with added EMG leads revealed few myoclonic jerks not associated with epileptiform abnormalities, ruling out seizure disorder, and her SSEP did not reveal giant potentials, thereby ruling out cortical myoclonus. Because of these results and the videotape evidence provided by the patient, we did not feel the need to do an EMG to further classify the myoclonus. A normal cervical and thoracic spine MRI ruled out any structural lesions as causes for these myoclonic movements. Based on these findings, we conclude that this represents a paraneoplastic phenomenon.

Propriospinal myoclonus has been reported before with breast cancer and also with mediastinal masses $[6,7]$. There have been no neurological paraneoplastic syndromes reported with papillary thyroid carcinoma; the only paraneoplastic syndrome reported with this neoplasm has been polymyalgia rheumatica [8].

Levetiracetam is an anticonvulsant well known to be efficacious in the treatment of different types of myoclonia [910] and improved our subject's myo- clonus, as did treating her primary malignancy.

In conclusion, this is the first reported case of propriospinal myoclonus as a paraneoplastic phenomenon with papillary thyroid carcinoma. Together with the cases referenced above, hopefully awareness will be raised among physicians confronted with proprionspinal or segmental myoclonus of the possibility of occult neoplasms, leading to timely and efficient treatment of these patients' malignancies.

\section{References}

1 Caviness JN, Brown P: Myoclonus: current concept and recent advances. Lancet Neurol 2004;3:598-607.

2 Brown P, Rothwell JC, Thompson PD, Marsden CD: Propriospinal myoclonus: evidence for spinal 'pattern' generation in humans. Mov Disord 1994;9:571-576.

3 Manconi M, Sferrazza B, Iannaccone S, Massimo A, Zucconi M: Ferini-Strambi L: Case of symptomatic propriospinal myoclonus evolving toward acute 'myoclonic status'. Mov Disord 2005;20:1646-1650.

4 Dropcho EJ: Update on paraneoplastic syndromes. Curr Opin Neurol 2005;18:331-336.
5 Pittock SJ, Lucchinetti CF, Parisi JE, et al: Amphiphysin autoimmunity: paraneoplastic accompaniments. Ann Neurol 2005;58: 96-107.

6 Salsano E, Ciano C, Romano S, Cornelio F, Di Donato S, Pareyson D: Propriospinal myoclonus with life threatening tonic spasms as paraneoplastic presentation of breast cancer. J Neurol Neurosurg Psychiatry 2006;77: 422-424.

7 Harada H, Hayashi A, Watanabe M, Tamaoka A, Shoji S: A case of stiff-man syndrome with head retraction like reflex myoclonus and jerky myoclonus of bilateral lower extremities which responded well to removal of mediastinal carcinoma (in Japanese). Rinsho Shinkeigaku 1999;39:1025-1028.

8 Tabata M, Kobayashi T: Polymyalgia rheumatica and thyroid papillary carcinoma. Intern Med 1994;33:41-44.

9 Krauss GL, Bergin A, Kramer RE, Cho YW, Reich SG: Suppression of post-hypoxic and post-encephalitic myoclonus with levetiracetam. Neurology 2001;56:411-412.

10 Keswani SC, Kossoff EH, Krauss GL, Hagerty C: Amelioration of spinal myoclonus with levetiracetam. J Neurol Neurosurg Psychiatry $2000 ; 73: 457-458$. 